\title{
Andrological and cytogenetic investigations of an infertile Przewalski's stallion
}

\section{Acta Veterinaria Hungarica}

69 (2021) 2, 189-193

DOI:

$10.1556 / 004.2021 .00027$

(c) 2021 The Author(s)

\section{RESEARCH ARTICLE}

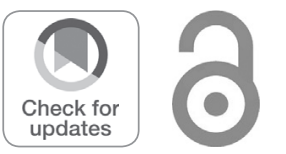

GABRIELLA KÚTVÖLGYI ${ }^{1^{*}}$, KRISTIN BRABENDER ${ }^{2}$, MAGNUS ANDERSSON ${ }^{3}$, ARIUNTUNGALAG JAVKHLAN ${ }^{4}$, SZABOLCS NAGY ${ }^{4}$, TAMÁS PÁBLE ${ }^{5}$, ISTVÁN EGERSZEGI ${ }^{6}$, ANDRÁS HIDAS ${ }^{6,7}$, ISTVÁN SOÓS ${ }^{8}$ and ANDRÁS KOVÁCS ${ }^{9}$

\footnotetext{
${ }^{1}$ Department of Precision Livestock Farming and Animal Biotechnics, Institute of Animal Sciences, Hungarian University of Agriculture and Life Sciences, Kaposvár Campus, Guba Sándor u. 40, H-7400 Kaposvár, Hungary

${ }^{2}$ Hortobágy National Park, Hungary

${ }^{3}$ Department of Production Animal Medicine, University of Helsinki, Helsinki, Finland

${ }^{4}$ Department of Precision Livestock Farming and Animal Biotechnics, Institute of Animal Sciences, Hungarian University of Agriculture and Life Sciences, Georgikon Campus, Keszthely, Hungary

${ }^{5}$ Vitafort Co. Ltd., Dabas, Hungary

${ }^{6}$ Department of Precision Livestock Farming and Animal Biotechnics, Institute of Animal Sciences, Hungarian University of Agriculture and Life Sciences, Gödöllő Campus, Gödöllö, Hungary

${ }^{7}$ Research Centre for Farm Animal Gene Conservation, National Centre for Biodiversity and Gene Conservation, Gödöllö, Hungary

${ }^{8}$ Al-Rakib Co. Ltd., Debrecen, Hungary

${ }^{9}$ Faculty of Agriculture and Food Sciences and Environmental Management, University of Debrecen, Hungary
}

Received: 13 May 2020 • Accepted: 30 June 2021

Published online: 22 July 2021

\begin{abstract}
The case of an 8-year-old, sexually active but infertile Przewalski's stallion (Equus ferus przewalskii) was studied. Besides the infertility, the stallion also showed permanent problems with its body condition, being obviously weaker than all the other group members. The horse was kept in a separate place for two years with 12 mares in its harem group (six mares had foals earlier); however, none of the mares covered got pregnant. Andrological and cytogenetic investigations revealed underdeveloped testes, arrested spermatogenesis, azoospermia, and $\mathrm{XY} / \mathrm{XXY} / \mathrm{X} 0$ mosaicism. We classify the case as a mosaic Klinefelter syndrome, the first reported case in Przewalski's horse.
\end{abstract}

\section{KEYWORDS}

Przewalski's horse, infertility, Klinefelter syndrome, mosaicism

\section{INTRODUCTION}

*Corresponding author. Tel.: +3620 9450178.

E-mail: kutvolgyi.gabriella@uni-mate. hu

\section{AKJournals}

The world's largest managed population of Przewalski's horses (Equus ferus przewalskii) lives under semi-wild conditions in the core zone of the Hortobágy National Park in Eastern Hungary in a reserve of 2,470 ha size. The population of the horses contained 295 (132 male, 163 female) individuals at the time of the investigation (October 2014), all of which were identifiable and genetically tested for parentage. The population goes back to 25 (8 male, 17 female) founders that had been chosen from different zoos and semi-reserves from all over Europe. The first harem group ( 1 male, 3 females) had been transported to the area in 1997. 
The development of the horse population is continuously monitored with the main focus on behavioural studies, health documentation and genetic patterns (Zimmermann et al., 2009). To avoid inbreeding and to ensure a higher genetic diversity in the population, genetically overrepresented stallions are culled each year. In 2014, eleven horses were culled for different reasons, namely chronic diseases, behavioural abnormalities, or high inbreeding factors. One of those horses, the stallion 'Gagarin', was known to be infertile with no sperm cells in his semen taken by electroejaculator (Christine Gohl, personal communication, 2011). Numerical sex chromosome abnormalities, including $\mathrm{X}$ polysomy in stallions, are not so rare in infertile domestic horses (Gluchovschi et al., 1970; Bouters et al., 1972; Fretz and Hare, 1976; Halnan et al., 1982; Kubien et al., 1993; Mäkinen et al., 2000; Iannuzzi et al., 2004); however, they have not yet been described in Przewalski's horse.

\section{CASE DESCRIPTION}

\section{Animal}

The stallion 'Gagarin' was born in Munich as a descendent of the highly inbred A-line. Fertility problems in both sexes are frequent in this line. Besides its infertility, the stallion also showed permanent problems with body condition, being obviously weaker than all the other group members. The horse arrived in Hortobágy in 2012. It was kept in a separate place for two years with 12 mares in his harem group (six mares had foals earlier); however, none of the mares covered got pregnant. To detect the cause of its infertility, andrological and cytogenetic investigations have been conducted.

\section{Methods}

Chromosome preparation. Blood samples were taken by syringes from the $\mathrm{v}$. jugularis from all 11 individuals (7 stallions, 1 gelding and 3 mares), including the stallion with impotentia generandi and filled into heparinised tubes (Vacuette $^{\circledR}$ Tube $6 \mathrm{~mL}$ LH Lithium Heparin, cat. no. 456088 (Greiner BioOne, Mosonmagyaróvár, Hungary) Lymphocyte cultures were established with our method elaborated for domestic horses (Chowdhary and Kovács, 1987). After 30-min sedimentation of the heparinised blood samples, $0.5 \mathrm{~mL}$ plasma containing leukocytes was added to $3.0 \mathrm{~mL}$ culture medium. The composition of the culture medium used is shown in Table 1. Lymphocyte culture, chromosome preparation and evaluation were carried out as described by Moorhead et al. (1960). The C-banding technique was carried out according to Sumner (1972).

Morphology and histology. The weight of the testes of 'Gagarin' was compared to that of five adult, healthy stallions. Testicular and epididymal structure was evaluated in histological sections. Sugar-lump-sized samples were collected and placed into sample containers filled with $10 \%$ neutral buffered formalin solution (Formaldehyde 4\%, cat.
Table 1. Composition of the medium used for lymphocyte culture

\begin{tabular}{lll}
\hline RPMI-1640 Medium & Sigma-Aldrich R8758 & $1,000 \mathrm{~mL}$
\end{tabular}

Fetal Bovine Serum

Phytohaemagglutinin

(PHA-P)

Pokeweed mitogen (PWM)

Glutamine-Penicillin-

Streptomycin solution

Sigma-Aldrich F4135 $200 \mathrm{~mL}$

Sigma-Aldrich L9017 $10 \mathrm{mg}$

Sigma-Aldrich L9379 $10 \mathrm{mg}$

Sigma-Aldrich G1146 $15 \mathrm{~mL}$

no. 9713.5000, VWR International Ltd, Radnor, Pennsylvania, USA). After embedding in paraffin, 3-4 $\mu \mathrm{m}$ thick slices were made from the tissue pieces. Histological sections were stained with haematoxylin and eosin staining technique, mounted by Canada balsam (SIAL-C1795, SigmaAldrich, St. Louis, Missouri, USA), then coverslipped and evaluated at $\times 400$ magnification using Leica DMRA light microscope (Leica Microsystems, Wetzlar, Germany).

Both caudae epididymides seemed to be empty; their content was collected by retrograde rinsing (Monteiro et al., 2011; Olaciregui et al., 2014). Suspensions were smeared and evaluated by Chicago sky blue and Giemsa viability and acrosome staining (Kútvölgyi et al., 2006).

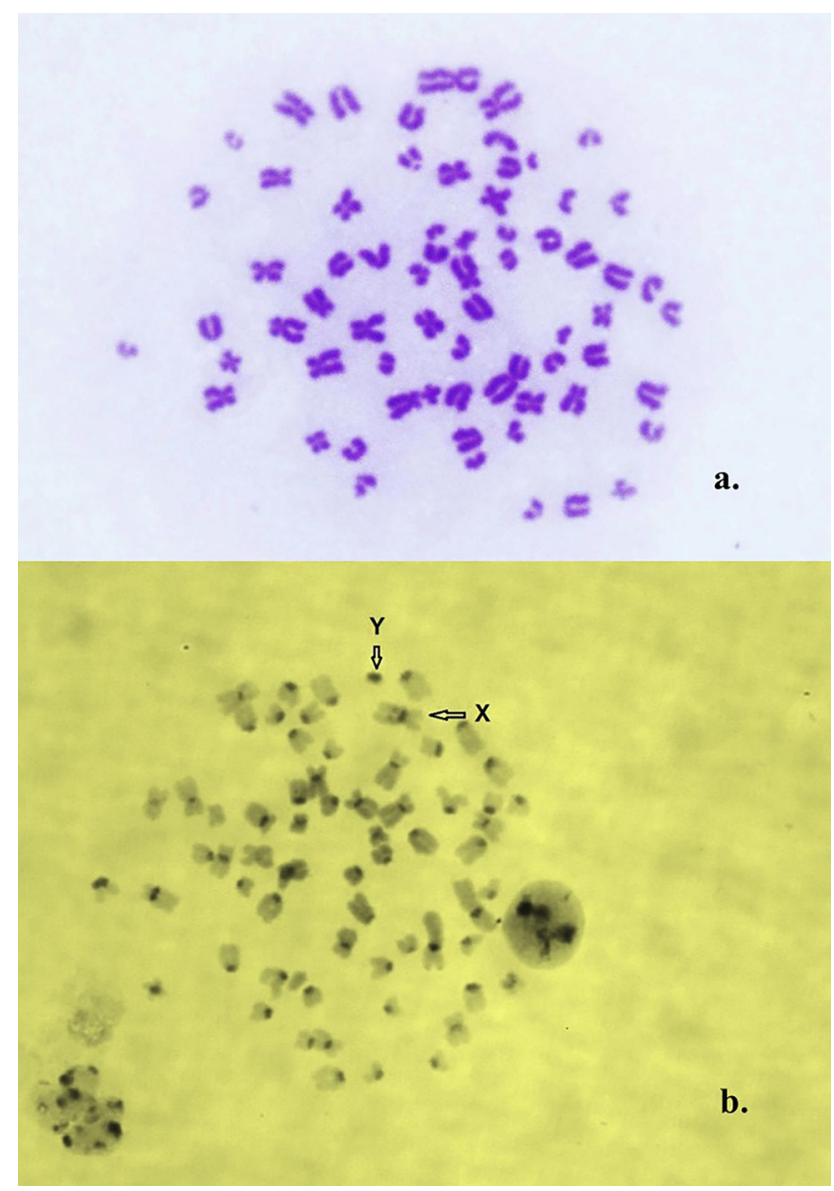

Fig. 1. $2 \mathrm{~N}=66, \mathrm{XY}$ karyotype, a: Giemsa staining; b: C-banding. The sex chromosomes are indicated with arrows 


\section{RESULTS}

In the preparations made from the lymphocyte cultures of the infertile stallion, three different cell lines containing 67, 66 and 65 chromosomes were found. In 73 out of 86 mitoses $(84.88 \%) 66$, in $7(8.14 \%) 67$, and in $6(6.98 \%) 65$ chromosomes were found.

$\mathrm{XY} / \mathrm{XXY} / \mathrm{X} 0$ mosaicism was diagnosed by the karyotypes of the XY, XXY, and $\mathrm{X} 0$ cell lines (Figs 1-3) and by the identification of both sex chromosomes: the $\mathrm{X}$ having a fine interstitial C-band on its long arm, and the $\mathrm{Y}$ being the smallest and darkest element (Matthews and Delhanty, 1979) (Figs 1 and 2).

The smaller body size of 'Gagarin' reminiscent of $\mathrm{XO}$ gonadal dysgenesis (human Turner syndrome) could be the only recognised consequence of the XO cell line.

The testicles of the horse were smaller than those of the other adult Przewalski's stallions examined (Table 2). The histology of the testes showed seminiferous tubules with arrested spermatogenesis at the spermatocyte/spermatid stage showing elongated spermatids as the most advanced stage in spermatogenesis (Figs 4 and 5).

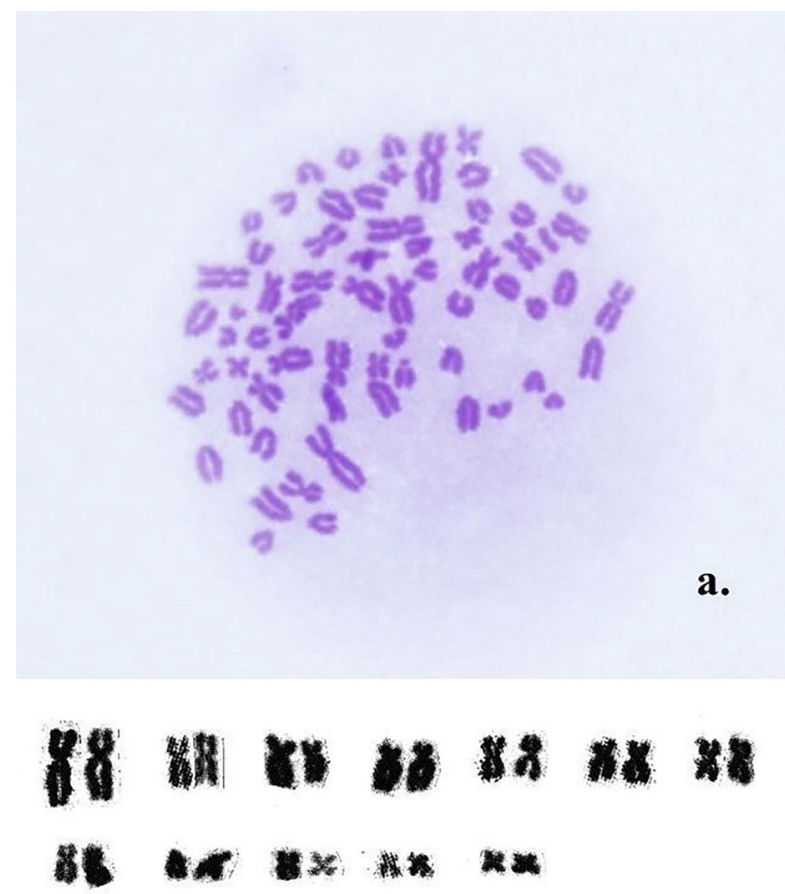

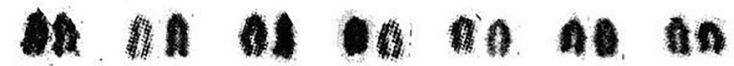

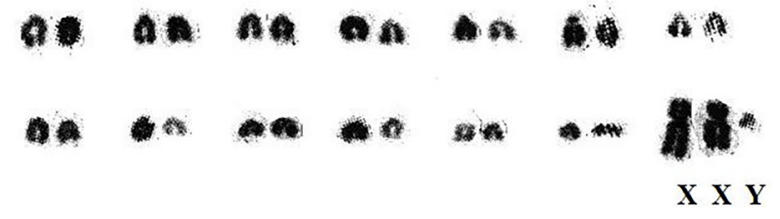

b.

Fig. $2.2 \mathrm{~N}=67$, $\mathrm{XXY}$ karyotype, a: Giemsa staining; b: karyogram

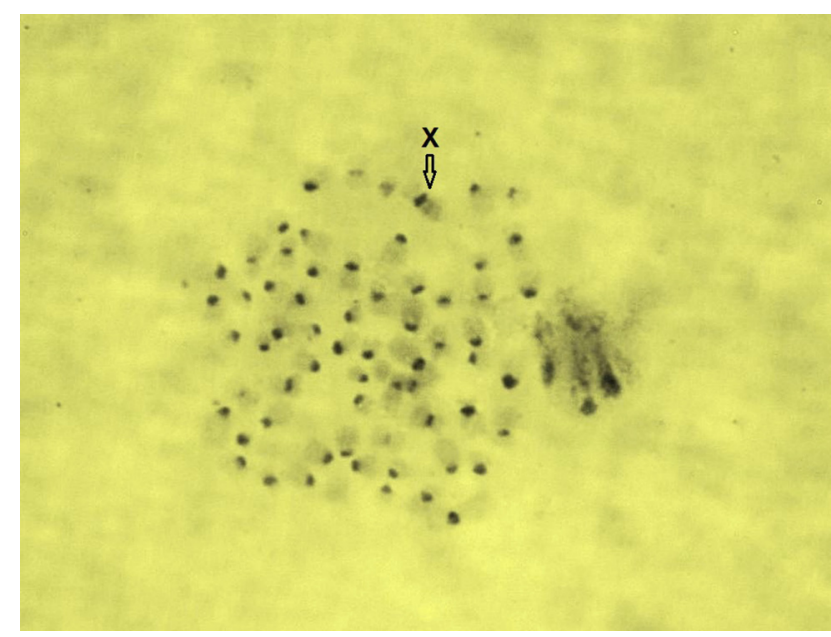

Fig. 3. $2 \mathrm{~N}=65$, X0 karyotype, C-banding. The $\mathrm{X}$-chromosome is indicated with an arrow

Table 2. Weight of the testes of the six adult stallions

\begin{tabular}{lcccc}
\hline Name & $\begin{array}{c}\text { Studbook } \\
\text { No. }\end{array}$ & $\begin{array}{c}\text { Age } \\
\text { (years) }\end{array}$ & $\begin{array}{c}\text { Left testis } \\
\text { weight }(\mathrm{g})\end{array}$ & $\begin{array}{c}\text { Right testis } \\
\text { weight }(\mathrm{g})\end{array}$ \\
\hline Gáspár & 4,415 & 10 & 131 & 150 \\
Ladomér & 5,454 & 5 & 167 & 182 \\
Ipoly & 4,730 & 8 & 193 & 175 \\
Fakó & 3,903 & 11 & 166 & 176 \\
Jánk & 4,995 & 7 & 161 & 164 \\
Gagarin & 5,324 & 8 & $\mathbf{1 1 0}$ & $\mathbf{1 0 9}$ \\
\hline
\end{tabular}

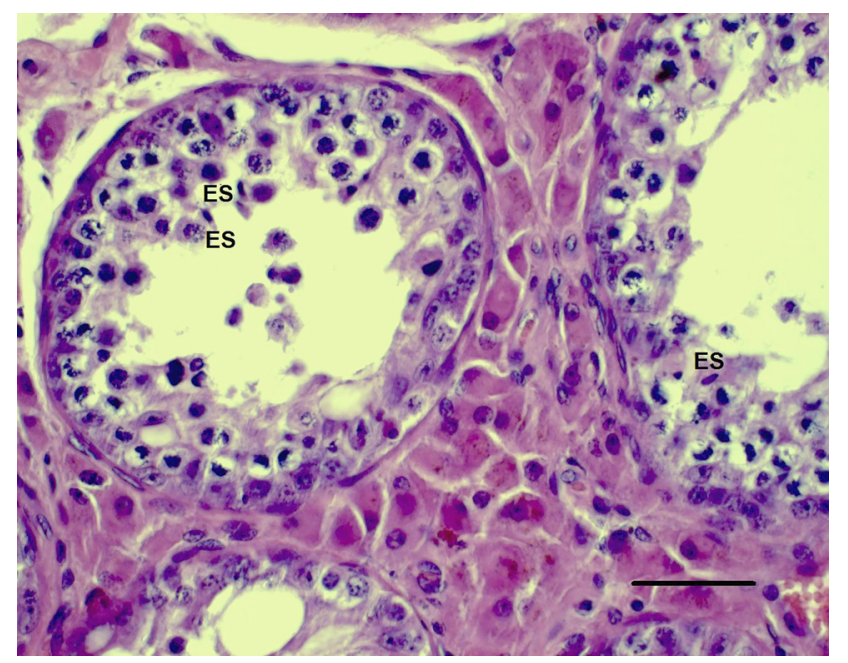

Fig. 4. Histological section of the stallion's left testicle. Haematoxylin and eosin (HE) staining, bar: $50 \mu \mathrm{m}$ : the most advanced stage of spermatogenesis is the elongated spermatid (ES)

We noticed the lack of normal spermatogenetic cycle in the seminiferous epithelium; the histological structure appeared to be disintegrated. Figure 6 shows several multinucleated cells in the lumen of the corpus epididymidis. Spermatozoa were not found in the tubules or in the lumen of the ductus epididymidis. 


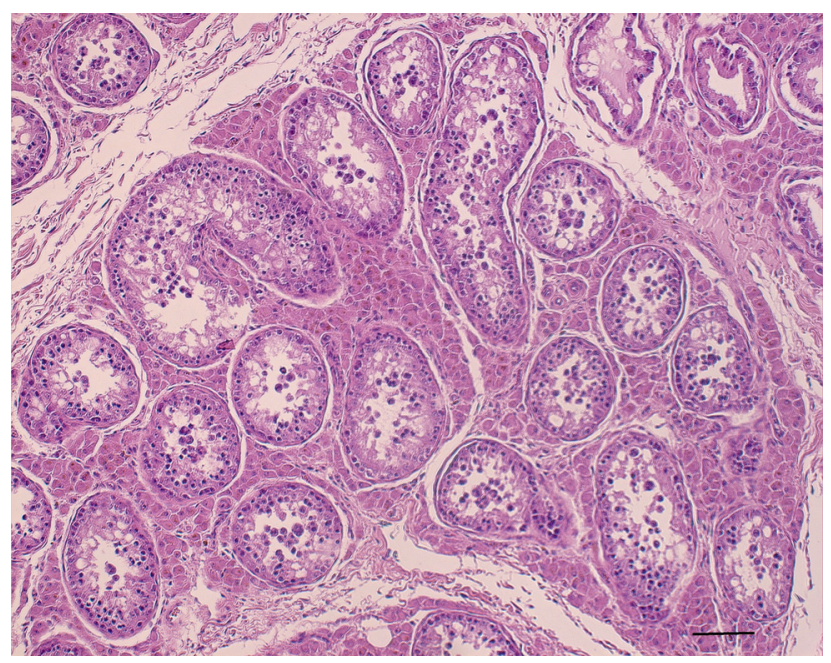

Fig. 5. Histological section of the stallion's right testicle (HE staining, bar: $100 \mu \mathrm{m}$ )

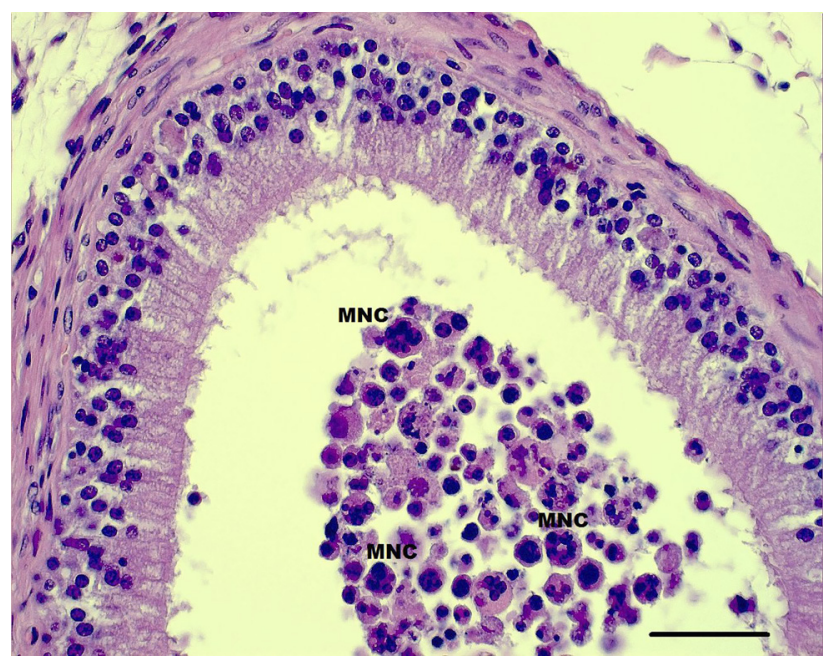

Fig. 6. Histological section of the stallion's right corpus epididymidis (HE staining, bar: $50 \mu \mathrm{m}$ ). MNC: multinucleated cell

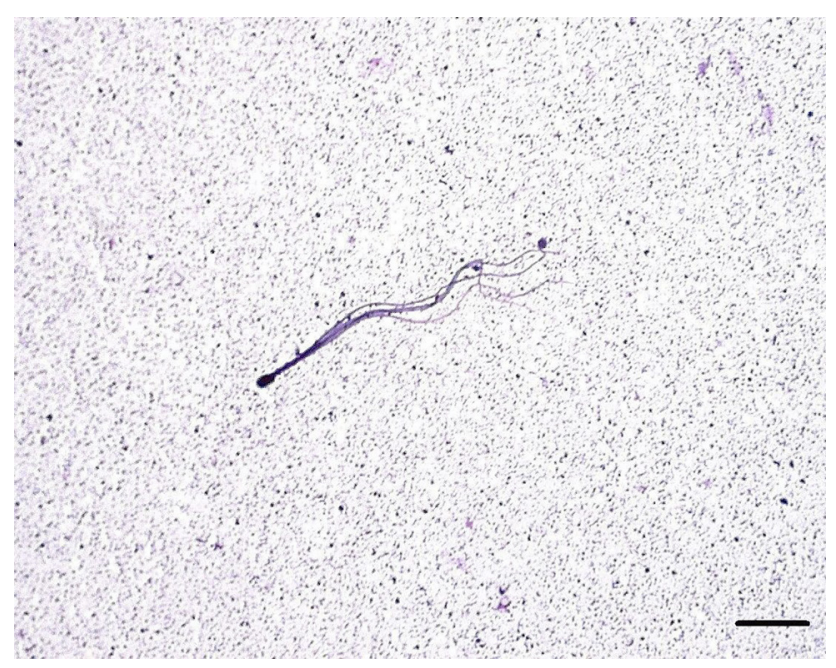

Fig. 7. Abnormal spermatozoon flushed from the cauda epididymidis (Bar: $10 \mu \mathrm{m}$ )
The tail of the epididymis appeared macroscopically empty. Azoospermia with very few malformed spermatozoa such as multi-tailed microcephalic cells and other head abnormalities were found in the smears (Fig. 7). There were no morphologically normal spermatozoa present.

\section{DISCUSSION}

We obtained satisfactory chromosome preparations after starting the blood cultures under extreme circumstances in the field. According to our experiences, the whole blood method is less convenient, as the red blood cells of Equidae and Felidae are extremely strongly coagulated by the lectins stimulating the lymphocytes.

Ten animals were successfully evaluated by chromosome analysis, nine having $2 \mathrm{~N}=66$ chromosomes as described by other authors (Benirschke et al., 1965; Matthews and Delhanty, 1979), while in the preparations made from the lymphocyte cultures of the infertile stallion, three different cell lines containing 66, 67 and 65 chromosomes were found.

The histological sections of the testes with incomplete meiosis and azoospermia/teratospermia (Blom, 1944) were similar to the XXXY and XXY cases, reported in domestic horses (Gluchovschi et al., 1970; Bouters et al., 1972; Fretz and Hare, 1976; Halnan et al., 1982; Kubien et al., 1993; Mäkinen et al., 2000; Iannuzzi et al., 2004) and other species, including the mosaic cases of human Klinefelter syndrome (Filadis et al., 1998; Wistuba 2010; Wikström and Dunkel, 2011; Samplaski et al., 2014). The XXY and X0 cell lines probably resulted from nondisjunction events in the mitoses of multipotent cells in the preimplantation embryo. We classify the present case as a mosaic Klinefelter syndrome, which is, to the best of our knowledge, the first reported case in Przewalski's horse.

\section{ACKNOWLEDGEMENTS}

We would like to express our thanks to Dr. Endre Felszeghy (Semmelweis University Faculty of Medicine, Institute of Foresic Medicine, Budapest) for the histological preparations. Special thanks to Dr. Waltraud Zimmermann, the coordinator of the breeding programme of Przewalski's horses for the opportunity to perform the analyses. Dr. A. Javkhlan received a scholarship from Tempus Public Foundation.

\section{REFERENCES}

Benirschke, K., Malouf, N., Low, R. J. and Heck, H. (1965): Chromosome complements: differences between Equus caballus and Equus przewalskii, Poljakoff. Science 148, 382-383.

Blom, E. (1944): On the occurrence in bull sperm of certain 'Medusa formations' derived from the epithelium of the efferent ducts of the testis. Acta Pathol. Microbiol. Scand. 21, 713-720. 
Bouters, R., Vandeplassche, M. and De Moor, A. (1972): An intersex (male pseudohermaphrodite) horse with 64XX/65XXY mosaicism. Equine Vet. J. 3, 150-153.

Chowdhary, B. and Kovács, A. (1987): Simple lymphocyte cultivation method for horse chromosome studies. J. Dairy Sci. 70 (Suppl. 1), 241.

Filadis, J. F., Syrrou, M. B., Bai, M. C., Georgiu J. A., Pagoulatos, G. N. and Giannakopoulos, X. (1998): Infertility and multiple urogenital abnormalities in a male with mosaic $46, \mathrm{XY} / 45, \mathrm{XO} /$ 47, XXY karyotype and mixed phenotype. Urol. Int. 61, 111114.

Fretz, P. B. and Hare, W. C. (1976): A male pseudohermaphrodite horse with 63Xo?/64XX/65XXY mixoploidy. Equine Vet. J. 8, 130-132.

Gluchovschi, N., Bistriceanu, M., Suciu, A. and Bratu, M. (1970): A case of intersexuality in the horse with type $2 \mathrm{~A}+\mathrm{XXXY}$ chromosome formula. British Vet. J. 126, 522-525.

Halnan, C. R. E., Watson, J. I. and Pryde, L. C. (1982): Detection by G- and C-band karyotyping of gonosome anomalies in horses of different breeds. J. Reprod. Fert. Suppl. 32, 627-628.

Iannuzzi, L., Di Meo, G. P., Perucatti, A., Spadetta, M., Incarnato, D., Parma, P., Iannuzzi, A., Ciotola, F., Peretti, V., Perrotta, G. and Di Palo, R. (2004): Clinical, cytogenetic and molecular studies on sterile stallion and mare affected by XXY and sex reversal syndromes, respectively. Caryologia 57, 400-404.

Kubien, E. M., Pozor, M. and Tischner, M. (1993): Clinical, cytogenetic and endocrine evaluation of a horse with $65, \mathrm{XXY}$ karyotype. Equine Vet. J. 24, 333-335.

Kútvölgyi, G., Stefler, J. and Kovács, A. (2006): Viability and acrosome staining of stallion spermatozoa by Chicago sky blue and Giemsa. Biotech. Histochem. 81, 109-117. Erratum (2007) in: Biotech. Histochem. 82, 45.

Matthews, J. G. and Delhanty, J. D. A. (1979): Chromosome studies in Przewalski horses (Equus przewalskii). In: de Boer, L. E. M. Bouman, J. G. and Bouman, I. (eds) Genetics and Hereditary
Diseases of the Przewalski Horse. Proceedings of the Arnhem Study-Conference organized by the Foundation for the Preservation and Protection of the Przewalski Horse, held on the 16th-17th of October, 1978 at the Burger's Zoo. Published by the Foundation for the Preservation and Protection of the Przewalski Horse, Rotterdam, The Netherlands. pp. 71-82.

Mäkinen, A., Katila, T., Andersson, M. and Gustavsson, I. (2000): Two sterile stallions with XXY-syndrome. Equine Vet. J. 32, 358-360.

Monteiro, G. A., Papa, F. O., Zahn, F. S., Dellaqua, J. A. , Jr., Melo, C. M., Maziero, R. R., Avanzi, B. R., Alvarenga, M. A. and Guasti, P. N. (2011): Cryopreservation and fertility of ejaculated and epididymal stallion sperm. Anim. Reprod. Sci. 127, 197201.

Moorhead, P. S., Nowell, P. C., Mellman, W. J., Battips, D. M. and Hungerford, D. A. (1960): Chromosome preparations of leukocytes cultured from human peripheral blood. Exp. Cell Res. 20, 613-616.

Olaciregui, M., Gil, L., Montón, A., Luño, V., Jerez, R. A. and Martí, J. I. (2014): Cryopreservation of epididymal stallion sperm. Cryobiology 68, 91-95.

Samplaski, M. K., Lo, K. C., Grober, E. D., Millar, A., Dimitropanolakis, A. and Jarvi, K. A. (2014): Phenotypic differences in mosaic Klinefelter patients as compared with non-mosaic Klinefelter patients. Fertil. Steril. 101, 950-955.

Sumner, A. T. (1972): A simple technique for demonstrating centromeric heterochromatin. Exp. Cell Res. 75, 304-306.

Wikström, A. M. and Dunkel, L. (2011): Klinefelter syndrome. Best Pract. Res. Clin. Endocrinol. Metab. 25, 239-250.

Wistuba, J. (2010): Animal models for Klinefelter's syndrome and their relevance for the clinic. Mol. Hum. Reprod. 16, 375-385.

Zimmermann, W., Brabender, K. and Kolter, L. (2009): A Przewalski's horse population in a Unique European Steppe Reserve - the Hortobágy National Park in Hungary. Equus, Zoo Praha 2009, 207-235. 